\title{
Trajectoires de groupes à 1-paramètre de quasi-isométries
}

Volker Mayer

\section{Introduction.}

Un homéomorphisme $g: \mathbb{R}^{2} \longrightarrow \mathbb{R}^{2}$ est une $(L$-)quasi-isométrie si pour tout $x, y \in \mathbb{R}^{2}$

$$
\frac{1}{L}\|x-y\| \leq\|g(x)-g(y)\| \leq L\|x-y\| .
$$

Soit $G=\left\{g_{t}: t \in \mathbb{R}\right\}$ un groupe à 1-paramètre. Il est dit quasiisométrique s'il existe $L \geq 1$ tel que tout élément de $\mathrm{G}$ est une $L$-quasiisométrie.

Le point de départ de ce travail est l'étonnant exemple de $\mathrm{P}$. Tukia [T2] d'un groupe quasi-isométrique du plan $\mathbb{R}^{2}$ n'étant pas quasiisométriquement conjugable à un groupe d'isométries, i.e. il ne s'écrit pas sous la forme

$$
G=f \circ \Phi \circ f^{-1},
$$

où $f: \mathbb{R}^{2} \longrightarrow \mathbb{R}^{2}$ est une quasi-isométrie et $\Phi$ est un groupe d'isométries. La raison pour laquelle ceci n'a pas lieu est simple. Le groupe $G$ de Tukia a une trajectoire $\Gamma=\left\{g_{t}(0): t \in \mathbb{R}\right\}$ le "snowflake" ou encore la courbe de Von Koch. Cette trajectoire $\Gamma$ n'est pas rectifiable; elle a même une dimension de Hausdorff strictement plus grande que un. 
Le fait qu'un groupe quasi-isométrique peut agir transitivement sur une courbe fractale est remarquable et la question se pose: quelles sont les trajectoires d'un groupe à 1-paramètre quasi-isométrique en général? On les appelle dans la suite quasi-isométrique cercle $(Q I$ cercle) ou, plus précisément, $L-Q I$-cercle si les éléments du groupe sont $L$-quasi-isométriques.

D. Sullivan [Su1] et P. Tukia [T1] ont montré qu'en dimension deux tout groupe quasiconforme est quasiconformément conjugué à un groupe conforme. Un groupe quasi-isométrique à 1-paramètre est alors le conjugué quasiconforme d'un groupe d'isométries à 1-paramètre parabolique ou elliptique; $c f$. la classification des applications conformes, par exemple dans Greenberg [4]. Par conséquent, les $Q I$-cercles sont des quasicercles, c'est à dire les images d'une droite (ou d'un cercle) par une application quasi-conforme de $\mathbb{R}^{2}$.

Avant d'énoncer des caractérisations des $Q I$-cercles fixons les notations necessaires. On note par $D(p, r)$ le disque ouvert centré en $p$ de rayon $r>0$. Dans tout le texte, disque signifie toujours disque ouvert. Le bord du disque $D(0,1)$ est noté $\mathbb{S}^{1}$. Le symbole $\Gamma(p, q)$ désigne un sous-arc demi-ouvert d'une courbe $\Gamma$, joignant $p$ à $q$ et contenant le point $p$ mais pas $q$. Quand $\Gamma$ est une courbe de Jordan on prend pour $\Gamma(p, q)$ le sous-arc avec le plus petit diamètre (s'il existe; sinon on choisit librement un des deux sous-arcs). Si $E \subset \Gamma$ est un sous-ensemble, $N(r, E)$ est le plus petit nombre de disques de rayon $r$ necessaires pour couvrir $E$.

Théorème 1.1. Une courbe $\Gamma$ est un $L$-QI-cercle si et seulement si une des propriétés suivantes est vérifiée.

(I) Il existe $M \geq 1$ et $h: \mathbb{R} \longrightarrow \Gamma$ ou $h: \mathbb{S}^{1} \longrightarrow \Gamma$ un homéomorphisme tel que

$$
\|h(x+t)-h(x)\| \leq M\|h(y+s)-h(y)\|,
$$

pour tous $x, y \in \mathbb{R}$ ou $\mathbb{S}^{1}$ et pour tout $0<t \leq s$.

(II) Il existe une mesure $\omega$ de $\Gamma$ non-triviale et $\sigma$-finie, i.e. $0<$ $\omega(\Gamma(p, q))<\infty$ pour tout $p, q \in \Gamma$ distincts, vérifiant pour une constante $A \geq 1$,

$$
\omega\left(\Gamma\left(p_{1}, p_{2}\right)\right) \leq A \omega\left(\Gamma\left(q_{1}, q_{2}\right)\right),
$$

pour tous $p_{i}, q_{i} \in \Gamma$ avec $\left\|p_{1}-p_{2}\right\|=\left\|q_{1}-q_{2}\right\|$. 
(III) $\Gamma$ vérifie la propriété géométrique suivante: il existe une constante $H \geq 1$ telle que, si $p_{1}, p_{2}, q_{1}, q_{2} \in \Gamma$ sont deux paires de points telles que $\left\|p_{1}-p_{2}\right\|=\left\|q_{1}-q_{2}\right\|$, alors

$$
N\left(r, \Gamma\left(p_{1}, p_{2}\right)\right) \leq H N\left(r, \Gamma\left(q_{1}, q_{2}\right)\right), \quad \text { pour tout } r>0 .
$$

En plus, toutes les constantes dépendent l'une de l'autre. Quand l'une d'entre elle est égale $\grave{a}$ un, les autres peuvent aussi être prises égales à un.

Le fait qu'un $Q I$-cercle doit être un quasicercle se reflète dans ce théorème. Si on remplace par exemple dans l'inégalité de (I) $y$ par $x$ on a précisement la condition qui dit que $h$ est une quasi-symétrie et donc que $\Gamma$ est un quasicercle. On va aussi voir que (II) aussi bien que la propriété géométrique (III) implique la propriété des trois points de Ahlfors: une courbe $\Gamma$ est un quasicercle s'il existe une constante $c \geq 1$ telle que $\operatorname{diam} \Gamma(p, q) \leq c\|p-q\|$.

Remarquons encore que si $\Gamma$ vérifie (II) ou (III) avec constante 1, alors $\Gamma$ est forcément une droite ou un cercle ( $c f$. Proposition 3.1).

Dans $[F M]$ K.J. Falconer et T.D. Marsh étudient les "quasi-selfsimilar" cercles. Grâce à leur étude on peut dire que ce sont des courbes de Jordan $\Gamma$ paramétrisables par un homéomorphisme $h: \mathbb{S}^{1} \longrightarrow \Gamma$ vérifiant

$$
\frac{\|x-y\|^{\alpha}}{c} \leq\|h(x)-h(y)\| \leq c\|x-y\|^{\alpha},
$$

pour tous $x, y \in \mathbb{S}^{1}$, où $c \geq 1$ et $1 / \alpha=\operatorname{Hdim}(\Gamma) \in[1,2[$. Ceci et le Théorème 1.1 impliquent que toutes ces courbes sont des exemples de $Q I$-cercles. En particulier les ensembles de Julia des fonctions $f_{\lambda}(z)=z^{2}+\lambda, \lambda$ appartenant à l'intérieur de la cardioïde principale de l'ensemble de Mandelbrot [Su2], aussi bien que les ensembles limites de certains groupes kleiniens [Bo].

Les "quasi-self-similar" cercles ont des jolies propriétés fractales. Par exemple, toutes les différentes dimensions (Box, Hausdorff) d'une telle courbe coïncident et, à une quasi-isométrie près, ils sont définis uniquement en fonction de leur dimension [FM]. Dans le Paragraphe 5 nous étudions comment étendre ces résultats aux $Q I$-cercles.

Je tiens à remercier Michel Zinsmeister pour des nombreuses discussions et pour m'avoir aidé à clarifier ce travail. Je remerci également 
le referee. La condition (II) du Théorème 1.1 est basée sur une idée à lui.

\section{2. Étude de la propriété géométrique.}

Nous résumons dans ce paragraphe quelques résultats importants pour la suite.

L'astuce suivante va être utilisée à plusieures reprises: il est clair que le nombre de disques de rayon $r>0$ necessaires pour pouvoir couvrir un disque $D(p, R), R \geq r$, se majore par une constante $\nu=\nu(R / r)$ dépendant que du rapport des rayons. Une conséquence immédiate de ceci est

$$
N(r, \Gamma(p, q)) \leq \nu\left(\frac{R}{r}\right) N(R, \Gamma(p, q)),
$$

quand $r \leq R$ et $p, q \in \Gamma$. Voyons pourquoi (II) et (III) sont des conditions plus fortes que la propriété des trois points de Ahlfors:

Lemme 2.1. Toute courbe $\Gamma$ vérifiant la propriété géométrique (III) est un $2 H$-quasicercle, i.e.

$$
\operatorname{diam} \Gamma(p, q) \leq 2 H\|p-q\|, \quad \text { pour tous } p, q \in \Gamma .
$$

Quand une courbe $\Gamma$ vérifie (II) elle est un $2 A$-quasicercle.

Preuve. Supposons le contraire dans le cas où $\Gamma$ vérifie (III): il existe $p, q \in \Gamma$ tels que diam $\Gamma(p, q)>2 H\|p-q\|$. Soit $r=\|p-q\|$. Il existe $a, b \in \Gamma(p, q)$ avec $\|a-b\|=r$ et $\|a-x\|<r$ pour tout $x \in \Gamma(a, b)$. D'où $N(r, \Gamma(a, b))=1$. Comme $\operatorname{diam} \Gamma(p, q)>2 H r$, le nombre $N(r, \Gamma(p, q))$ est strictement plus grand que $H$. On a alors une contradiction avec (III).

Considérons maintenant une courbe $\Gamma$ vérifiant (II). Soient $p, q \in \Gamma$ et $a, b \in \overline{\Gamma(p, q)}$ tel que $\operatorname{diam} \Gamma(p, q)=\|a-b\|$. On choisi $m \in \mathbb{N}$ de sorte que $(m+1)\|p-q\| \geq\|a-b\| \geq m\|p-q\|$. Alors, par (II), $\omega(\Gamma(a, b)) \geq m \omega(\Gamma(p, q)) / A$. Or, $\Gamma(a, b) \subset \Gamma(p, q)$, et donc $m \leq A$. D'où, $\operatorname{diam} \Gamma(p, q) \leq(m+1)\|p-q\| \leq 2 A\|p-q\|$.

Les nombres $N(r, \gamma)$ sont définis dans l'introduction. Nous aurons besoin d'une quantité analogue: on appelle $r$-ensemble de $\Gamma(p, q)$ un ensemble de points $\left\{x_{0}=p, x_{1}, \ldots, x_{n}=q\right\} \subset \overline{\Gamma(p, q)}$ ordonné et maximal pour que $\left\|x_{i-1}-x_{i}\right\|=r, i=1,2, \ldots, n-1$. On note $n(r, \Gamma(p, q))=n$. 
Lemme 2.2. Pour un $K$-quasicercle $\Gamma$ les nombres $N(r, \Gamma(p, q))$ et $n(r, \Gamma(p, q))$ sont équivalents:

$$
N(r, \Gamma(p, q)) \leq n(r, \Gamma(p, q)) \leq \nu(4 K) N(r, \Gamma(p, q)) .
$$

Preuve. Choisissons un ensemble $\left\{x_{0}, \ldots, x_{n}\right\} \subset \bar{\gamma}, \gamma=\Gamma(p, q)$, maximal pour la condition suivante: on prend $x_{0}=p$ et successivement $x_{i} \in \Gamma\left(x_{i-1}, q\right), i=1, \ldots, n-1$, de sorte que $\left\|x_{i-1}-x_{i}\right\|=r$ et $\left\|x-x_{i-1}\right\|<r$ pour tout $x \in \Gamma\left(x_{i-1}, x_{i}\right)$. Enfin on prend comme dernier point $x_{n}=q$. Ce choix assure que les disques $D\left(x_{i}, r\right), i=0,1, \ldots, n-1$ couvrent $\gamma$. D'où $N(r, \gamma) \leq n \leq n(r, \gamma)$.

Partons maintenant d'un $r$-ensemble $\left\{x_{0}, x_{1}, \ldots, x_{n}\right\}$ de $\gamma=\Gamma(p, q)$. Quand $n=1$ tout est clair. Soit alors $n \geq 2$. Dans $\gamma_{i}=\Gamma\left(x_{i-1}, x_{i}\right)$, $i=1, \ldots, n-1$, il existe un point $p_{i}$ tel que $\min \left\{\left\|p_{i}-x_{i-1}\right\|,\left\|p_{i}-x_{i}\right\|\right\} \geq$ $r / 2$. $\Gamma$ étant un $K$-quasicercle, $\left\|x-p_{i}\right\| \geq r / 2 K$ pour tout $x \in \gamma \backslash \gamma_{i}$. Un disque de rayon $r / 4 K$ contenant un des points $p_{i}$ n'a pas de point en commun avec $\gamma \backslash \gamma_{i}$. D'où et par (2.2) on a bien

$$
n(r, \gamma) \leq N\left(\frac{r}{4 K}, \gamma\right) \leq \nu(4 K) N(r, \gamma)
$$

Lemme 2.3. Soit $\Gamma$ une courbe vérifiant (III). Alors, il existe une constante $\eta(H)>0$ dépendant que de $H$ telle que pour tout $p, q \in \Gamma$ et $0<r \leq R \leq\|p-q\|$ ainsi que $a, b \in \Gamma$ avec $\|a-b\|=R$ on a

$$
\begin{aligned}
\eta(H) n(R, \Gamma(p, q)) N(r, \Gamma(a, b)) & \leq N(r, \Gamma(p, q)) \\
& \leq H n(R, \Gamma(p, q)) N(r, \Gamma(a, b)) .
\end{aligned}
$$

Preuve. Notons $\gamma=\Gamma(p, q),\left\{x_{0}, \ldots, x_{n}\right\}$ un $R$-ensemble de $\gamma, \gamma_{i}=$ $\Gamma\left(x_{i-1}, x_{i}\right)$ et soit $a, b \in \Gamma$ avec $\|a-b\|=R$. Quand $n=n(R, \gamma)=1$ necessairement $\|p-q\|=\|a-b\|$ et le lemme suit directement de (III). Supposons alors $n \geq 2$. A cause de (III) on a

$$
N(r, \gamma) \leq \sum_{i=1}^{n} N\left(r, \gamma_{i}\right) \leq H n N(r, \Gamma(a, b)) .
$$

Pour voir l'autre inégalité considérons d'abord le cas $r \leq R / 4 H$. Cette restriction fait qu'un disque $D(x, r)$ a une intersection non vide 
avec au plus deux des arcs $\gamma_{i}$ qui doivent être des arcs voisins. Sinon il existe $u, v \in \Gamma(p, q)$ avec $\|u-v\|<2 r$ et tel que $\gamma_{i} \subset \Gamma(u, v)$ pour un $i \in\{2, \ldots, n-1\}$. Or, $\Gamma$ est un $2 H$-quasicercle et donc

$$
\|u-v\| \geq \frac{1}{2 H} \operatorname{diam} \Gamma(u, v) \geq \frac{1}{2 H} R \geq 2 r .
$$

Il est alors possible d'extraire d'un recouvrement minimal de $\Gamma(p, q)$ de disques de rayon $r$ un recouvrement de $\bigcup_{1 \leq 2 k+1 \leq n} \gamma_{2 k+1}$ tel que chaque disque a une intersection non vide avec qu'un seul arc $\gamma_{2 k+1}$. D'où, $N(r, \Gamma(p, q)) \geq \sum_{1 \leq 2 k+1 \leq n} N\left(r, \gamma_{2 k+1}\right)$. Le même raisonnement s'applique aux $\gamma_{i}$ d'indice pair, ce qui implique

$$
\begin{aligned}
2 N(r, \Gamma(p, q)) & \geq \sum_{i=1}^{n} N\left(r, \gamma_{i}\right) \\
& \geq \frac{1}{H}(n-1) N(r, \Gamma(a, b)) \\
& \geq \frac{1}{2 H} n(R, \gamma) N(r, \Gamma(a, b)) .
\end{aligned}
$$

Il reste à voir le cas $R \geq r>R / 4 H$. Par les lemmes précédents et $\operatorname{avec}(2.2)$ on voit que

$$
N(r, \Gamma(a, b)) \leq N\left(\frac{R}{4 H}, \Gamma(a, b)\right) \leq \nu\left(8 H^{2}\right) N(2 H R, \Gamma(a, b))=\nu\left(8 H^{2}\right)
$$

et

$$
n(R, \Gamma(p, q)) \leq \nu(8 H) N(R, \Gamma(p, q)) \leq \nu(8 H) N(r, \Gamma(p, q)) .
$$

La constante $\eta(H)=\min \left\{1 / 4 H, 1 / \nu(8 H) \nu\left(8 H^{2}\right)\right\}$ vérifie alors l'inégalité de gauche dans les deux cas.

Lemme 2.4. Soit $\Gamma$ une courbe vérifiant (III). Alors, pour toute constante $\tilde{H} \geq 1$ il existe $d>0$ dépendant que de $\tilde{H}$ et de la constante $H$ de (III) telle que si $N\left(r, \Gamma\left(p_{1}, p_{2}\right)\right) \leq \tilde{H} N\left(r, \Gamma\left(q_{1}, q_{2}\right)\right)$ pour un $r>0$ avec $r \leq\left\|q_{1}-q_{2}\right\|$ alors $\left\|p_{1}-p_{2}\right\| \leq d\left\|q_{1}-q_{2}\right\|$.

Preuve. Soit $\left\|p_{1}-p_{2}\right\|>\left\|q_{1}-q_{2}\right\|=R$. Par le Lemme 2.3 on a

$$
N\left(r, \Gamma\left(p_{1}, p_{2}\right)\right) \geq \eta(H) n\left(R, \Gamma\left(p_{1}, p_{2}\right)\right) N\left(r, \Gamma\left(q_{1}, q_{2}\right)\right) \text {. }
$$


En appliquant l'hypothèse on en déduit $\tilde{H} / \eta(H) \geq n\left(R, \Gamma\left(p_{1}, p_{2}\right)\right)$, ce qui montre $\left\|p_{1}-p_{2}\right\| \leq d\left\|q_{1}-q_{2}\right\|$ avec $d=\tilde{H} / \eta(H)$.

\section{Démonstration du Théorème 1.1 .}

(I) implique $\Gamma$ est un $Q I$-cercle. Cette preuve est fortement basée sur un argument utilisé par Tukia dans [T2].

a) Le cas non compact; on suppose que $\Gamma$ est homéomorphe à $\mathbb{R}$. On cherche un groupe quasi-isométrique à 1-paramètre $G=\left\{g_{t}: t \in \mathbb{R}\right\}$ pour lequel $\Gamma$ est une trajectoire: $\Gamma=\left\{g_{t}(p): t \in \mathbb{R}\right\}$ avec $p \in \Gamma$ un point quelconque.

Soit $h: \mathbb{R} \longrightarrow \Gamma$ une paramétrisation vérifiant (I) et notons par $H: \mathbb{R}^{2} \longrightarrow \mathbb{R}^{2}$ l'extension $K=K(M)$-quasiconforme de BeurlingAhlfors-Tukia [T3] de $h$. On considére le groupe

$$
G=H \circ\{x \longrightarrow x+t: t \in \mathbb{R}\} \circ H^{-1}=H \circ T \circ H^{-1} .
$$

Ce groupe a $\Gamma$ comme trajectoire. Afin de montrer que $G$ est un groupe quasi-isométrique on va d'abord établir l'inégalité

$$
\frac{\operatorname{dist}\left\{H\left(u_{1}, v\right), \Gamma\right\}}{\operatorname{dist}\left\{H\left(u_{2}, v\right), \Gamma\right\}} \leq L_{1}
$$

pour tous $u_{1}, u_{2} \in \mathbb{R}$ et $v \in \mathbb{R} \backslash\{0\}$, pour une constante $L_{1}=L_{1}(M) \geq 1$.

Il existe $t_{i} \in \mathbb{R}$ avec dist $\left\{H\left(u_{i}, v\right), \Gamma\right\}=\left\|H\left(u_{i}, v\right)-h\left(t_{i}\right)\right\|, i=1,2$. Soit $s_{1} \in \mathbb{R}$ tel que

$$
\left\|\left(u_{1}, v\right)-\left(s_{1}, 0\right)\right\|=\left\|\left(u_{2}, v\right)-\left(t_{2}, 0\right)\right\|=l
$$

et utilisons le fait qu'une application quasiconforme de $\mathbb{R}^{2}$ est $K^{\prime}$ quasisymétrique avec $K^{\prime}=K^{\prime}\left(K^{\prime}\right)$ est une constante dépendant que de $K$ et donc que de $\mathrm{M}$, [Ge2]. Alors,

$$
\operatorname{dist}\left\{H\left(u_{1}, v\right), \Gamma\right\} \leq\left\|H\left(u_{1}, v\right)-h\left(s_{1}\right)\right\| \leq K^{\prime}\left\|h\left(s_{1}+l\right)-h\left(s_{1}\right)\right\|
$$

et donc, avec (I),

$$
\begin{aligned}
\operatorname{dist}\left\{H\left(u_{1}, v\right), \Gamma\right\} & \leq K^{\prime} M\left\|h\left(t_{2}+l\right)-h\left(t_{2}\right)\right\| \\
& \leq K^{\prime 2} M\left\|H\left(u_{2}, v\right)-h\left(t_{2}\right)\right\| .
\end{aligned}
$$


Par le choix de $t_{2}$, dist $\left\{H\left(u_{1}, v\right), \Gamma\right\} \leq K^{\prime 2} M \operatorname{dist}\left\{H\left(u_{2}, v\right), \Gamma\right\}$ ce qui est précisément (3.3) avec $L_{1}=K^{\prime 2} M$.

On conclut maintenant comme dans [T2] preuve du Lemme 1. Soient $U_{1}=H\left(\left\{x_{2}>0\right\}\right)$ et $U_{2}=H\left(\left\{x_{2}<0\right\}\right)$ les composantes connexes de $\mathbb{R}^{2} \backslash \Gamma$. L'application $H$, étant l'extension de Beurling-AhlforsTukia de $h$, est, restreinte à $\left\{x_{2}>0\right\}$ ou à $\left\{x_{2}<0\right\}$, difféomorphe et une quasi-isométrie hyperbolique. La constante de quasi-isométrie hyperbolique dépend aussi que de $M$ [T3]. On définit sur $U_{j}$ la métrique quasi-hyperbolique par $|d y| / \operatorname{dist}\{y, \Gamma\}$. Cette métrique étant équivalente à la métrique hyperbolique, il existe $L_{2}=L_{2}(M) \geq 1$ tel que

$$
\frac{1}{L_{2}} \frac{|d y|}{\operatorname{dist}\{y, \Gamma\}} \leq \frac{\left|d g_{t}(y)\right|}{\operatorname{dist}\left\{g_{t}(y), \Gamma\right\}} \leq L_{2} \frac{|d y|}{\operatorname{dist}\{y, \Gamma\}}
$$

pour tout $t \in \mathbb{R}$ et $y \in U_{j}, j=1,2$. Or, $y$ et $g_{t}(y)$ sont dans une même courbe $H\left(\left\{\left(x_{1}, v\right): x_{1} \in \mathbb{R}\right\}\right)$ pour un $v \neq 0$. L'inégalité (3.3) implique donc

$$
\frac{1}{L_{1} L_{2}}|d y| \leq\left|d g_{t}(y)\right| \leq L_{1} L_{2}|d y|, \quad \text { pour presque tout } y \in \mathbb{R}^{2} .
$$

Les $g_{t}$ étant quasiconformes on en déduit que le groupe $G$ est bien un groupe quasi-isométrique (pour la métrique euclidienne) et que sa constante bilipschitzienne dépend que de $M$.

b) Le cas compact. On suppose maintenant qu'il existe $h: \mathbb{S}^{1} \longrightarrow \Gamma$ vérifiant (I). Soit encore $H$ l'extension de Beurling-Ahlfors-Tukia de $h$ et $G=H \circ \mathcal{R} \circ H^{-1}$ où $\mathcal{R}=\{x \longmapsto U x: U$ matrice orthogonale $\}$ le groupe des rotations euclidiennes fixant 0 et $\infty$. Ce groupe a $\Gamma$ comme trajectoire. Pour montrer que $G$ est un groupe quasi-isométrique on peut supposer, quitte à conjuguer $G$ avec une translation, que 0 est un point fixe des éléments de ce groupe. Supposons pour l'instant aussi qu'il existe $p \in \Gamma$ avec $\|p\|=1$. Ceci implique $\Gamma \subset A\left(K^{\prime}\right)=\left\{x \in \mathbb{R}^{2}\right.$ : $\left.1 / K^{\prime} \leq\|x\| \leq K^{\prime}\right\}$ puisque l'application $H$ est $K^{\prime}$-quasisymétrique avec $K^{\prime}$ dépendant que de $M$ et on a $H(0)=0$; le groupe $G$ fixe 0 .

Notons $d_{\mathbb{S}}$ la métrique sphérique (définie par $\|d x\| /\left(1+\|x\|^{2}\right)$ ). L'involution $i(x)=x /\|x\|^{2}$ est une isométrie pour cette métrique. La métrique euclidienne et la métrique sphérique étant équivalentes dans $A\left(K^{\prime}\right)$, l'application $\bar{h}=i \circ h: \mathbb{S}^{1} \longrightarrow \bar{\Gamma}=i(\Gamma)$ vérifie aussi l'inégalité de (I) pour une constante $\bar{M}$ dépendant que de $M$.

Soit $C_{1}$ la composante connexe de $\mathbb{R}^{2} \backslash \Gamma$ contenant 0 et $C_{2}$ l'autre. On considère $G_{1}=\left.G\right|_{C_{1}}$ et $\bar{G}_{2}=\left.i \circ G\right|_{C_{2}} \circ i$. Comme dans le cas 
non compact on montre que $G_{1}$ et $\bar{G}_{2}$ sont quasi-isométriques -pour le groupe $\bar{G}_{2}$ on utilise que $\bar{h}$ vérifi (I) et que $\bar{H}=i \circ H \circ i$ est une extension quasiconforme de $\bar{h}$ ayant les propriétés de l'extension de Beurling-Ahlfors-Tukia.

Dans $C_{1} \cup i\left(C_{2}\right) \subset D\left(0, K^{\prime}\right)$ la métrique euclidienne et la métrique sphérique sont équivalentes. $G_{1}, \bar{G}_{2}$ et donc aussi $G$ sont alors des groupes quasi-isométriques pour la métrique sphérique. Or, un groupe de $\mathbb{R}^{2}$ dont tout élément fixe 0 est quasi-isométrique pour la métrique sphérique si et seulement s'il l'est pour la métrique euclidienne ( $c f$. [T3], on peut le voir en utilisant l'expression infinitesimale de cette métrique). $G$ est alors bien un groupe quasi-isométrique pour la métrique euclidienne et sa constante bilipschitzienne est contrôlé par $M$.

Quand aucun point $p \in \Gamma$ est à distance un de l'origine, le groupe $G^{*}=d_{1 / r} \circ G \circ d_{r}$, avec $d_{r}(x)=r x$ et $r=\|p\|$ pour un $p \in \Gamma$ quelconque, est, par le précédent raisonnement, un groupe quasi-isométrique dont la constante bilipschitzienne dépend que de $M$. Il en est alors de même pour $G$ puisque conjugaison d'un groupe quasi-isométrique par similitude ne change pas sa constante bilipschitzienne.

$\Gamma$ est un $Q I$-cercle implique (III). Il existe $G=\left\{g_{t}: t \in \mathbb{R}\right\}$ un groupe $L$-quasi-isométrique de $\mathbb{R}^{2}$ agissant transitivement sur $\Gamma$. La courbe $\Gamma$ est un $K(L)$-quasicercle puisque $G$ se conjugue quasiconformément en un groupe isométrique et on a un contrôle de la dilatation de l'application conjugant en fonction de $L$, voir [T1].

Soient $\gamma_{1}=\Gamma\left(p_{1}, p_{2}\right)$ et $\gamma_{2}=\Gamma\left(q_{1}, q_{2}\right)$ des sous-arcs de $\Gamma$ avec $R=\left\|p_{1}-p_{2}\right\|=\left\|q_{1}-q_{2}\right\|$. On veut montrer $N\left(r, \gamma_{2}\right) \leq H N\left(r, \gamma_{1}\right)$ avec $H=H(L)$. Pour éviter un problème d'orientation on suppose que soit $g_{t}\left(\gamma_{1}\right) \subset \gamma_{2}$ soit $g_{t}\left(\gamma_{1}\right) \supset \gamma_{2}$ où $t \in \mathbb{R}$ tel que $g_{t}\left(p_{1}\right)=q_{1}$.

On prend $\left\{x_{0}=q_{1}, x_{1}, \ldots, x_{m}\right\}$ ordonné et maximal de sorte que $x_{i} \in \gamma_{2}$ pour $i=0,1, \ldots, m-1$ et tel que $\left\|x_{i}-x_{i-1}\right\|=R / L$ ainsi que $\left\|x-x_{i-1}\right\|<R / L$ quand $x \in \Gamma\left(x_{i-1}, x_{i}\right)$. Le choix de ces points et le Lemme 2.2 impliquent $m \leq n\left(R / L, \gamma_{2}\right) \leq \nu(4 K) N\left(R / L, \gamma_{2}\right)$. $\Gamma$ est un $K$-quasicercle et donc $\gamma_{2} \subset D\left(q_{1}, K R\right)$. Avec (2.2) on en déduit $m \leq \nu(4 K) \nu(L K)=H_{1}$.

Pour conclure on majore les nombres $N\left(r, \Gamma\left(x_{i-1}, x_{i}\right)\right)$ en fonction de $N\left(r, \gamma_{1}\right)$. Soit $t \in \mathbb{R}$ tel que $g_{t}\left(p_{1}\right)=x_{i-1}$. Par le choix des $x_{i}$ et le fait que le groupe est $L$-quasi-isométrique $\Gamma\left(x_{i-1}, x_{i}\right) \subset g_{t}\left(\gamma_{1}\right)$. Prenons $D_{j}=D\left(y_{j}, r\right), j=1, \ldots, N$ un recouvrement minimal de $\gamma_{1}$. Or, $g_{t}\left(D_{j}\right) \subset D\left(g_{t}\left(y_{j}\right), L r\right)$ et donc, encore avec (2.2), on a

$$
\frac{1}{\nu(L)} N\left(r, \Gamma\left(x_{i-1}, x_{i}\right)\right) \leq N\left(L r, \Gamma\left(x_{i-1}, x_{i}\right)\right) \leq N\left(r, \gamma_{1}\right) .
$$


D'où

$$
N\left(r, \gamma_{2}\right) \leq \sum_{i=1}^{m} N\left(r, \Gamma\left(x_{i-1}, x_{i}\right)\right) \leq \nu(L) H_{1} N\left(r, \gamma_{1}\right)
$$

ce qui termine la preuve. Remarquons que $H=H_{1} \nu(L)$ dépend que de $L$.

(III) implique (I). Par le Lemme 2.1 une courbe vérifiant (III) est un quasicercle. Elle est alors soit homéomorphe à $\mathbb{R}$ soit à $\mathbb{S}^{1}$.

a) Le cas non compact. Supposons d'abord que $\Gamma$ est une courbe vérifiant (III) et homéomorphe à $\mathbb{R}$. On peut supposer que $0 \in \Gamma$. Notons $\Gamma^{+}$l'une des composantes connexes de $\Gamma \backslash\{0\}$ et soit $\gamma=\Gamma(0, q) \subset$ $\bar{\Gamma}^{+}$choisi de sorte que $\|q\|=1$.

Considérons un $m \in \mathbb{N}$ tel qu'il existe un $r_{m}>0$ avec $N\left(r_{m}, \gamma\right)=$ $m$. A ce $m$ on associe une application continue $h_{m}: \mathbb{R} \longrightarrow \mathbb{R}^{2}$ de la façon suivante: soit $\left\{p_{i} \in \Gamma: i \in \mathbb{Z}\right\}$ un ensemble de points ordonnés avec $p_{0}=0, p_{1} \in \Gamma^{+}$et $\left\|p_{i}-p_{i-1}\right\|=r_{m}$ pour tout $i \in \mathbb{Z}$. L'application $h_{m}$ est alors définie par $h(i / m)=p_{i}$ et par

$$
\left.h_{m}\right|_{[(i-1) / m, i / m]}:\left[\frac{i-1}{m}, \frac{i}{m}\right] \longrightarrow\left[p_{i-1}, p_{i}\right] \text { affine. }
$$

On montre que $\left\{h_{m}\right\}$ est equicontinue et que la limite d'une sous-suite convergente est bien un homéomorphisme de $\mathbb{R}$ sur $\Gamma$ satisfaisant (I). La preuve de ceci est basée sur les inégalités suivantes: il existe $C_{1}(H)$ tel que

$$
\left\|h_{m}\left(\frac{i+k}{m}\right)-h_{m}\left(\frac{i}{m}\right)\right\| \leq C_{1}(H)\left\|h_{m}\left(\frac{j+l}{m}\right)-h_{m}\left(\frac{j}{m}\right)\right\|,
$$

pour tous $i, j \in \mathbb{Z}$ et $0<k \leq l$, et il existe $C_{2}(H)$ tel que

$$
\left\|h_{M}\left(\frac{i}{M}\right)\right\| \leq C_{2}(H)\left\|h_{m}\left(\frac{1}{m}\right)\right\|,
$$

quand $M \geq m$ et $i / M \leq 1 / m$.

Montrons (3.4). $\Gamma$ étant un $2 H$-quasicercle

$$
\left\|p_{j+l}-p_{j}\right\| \geq \frac{\operatorname{diam} \Gamma\left(p_{j}, p_{j+l}\right)}{2 H} \geq \frac{r_{m}}{2 H}
$$

L'inégalité (3.4) est alors une conséquence du Lemme 2.4 s'il existe $\tilde{H}$ dépendant que de $\mathrm{H}$ tel que

$$
N\left(\frac{r_{m}}{2 H}, \Gamma\left(p_{i}, p_{i+k}\right)\right) \leq \tilde{H} N\left(\frac{r_{m}}{2 H}, \Gamma\left(p_{j}, p_{j+l}\right)\right)
$$


Les disques $D\left(p_{i+\nu}, 2 H r_{m}\right), \nu=1, \ldots, k$, couvrent $\Gamma\left(p_{i}, p_{i+k}\right)$. De ceci et de (2.2) on tire

$$
N\left(\frac{r_{m}}{2 H}, \Gamma\left(p_{i}, p_{i+k}\right)\right) \leq \nu\left(4 H^{2}\right) N\left(2 H r_{m}, \Gamma\left(p_{i}, p_{i+k}\right)\right) \leq \nu\left(4 H^{2}\right) k
$$

D'autre part, $l \leq n\left(r_{m}, \Gamma\left(p_{j}, p_{j+l}\right)\right)$ puisque $\left\{p_{j}, \ldots, p_{j+l}\right\}$ est un $r_{m^{-}}$ ensemble de $\Gamma\left(p_{j}, p_{j+l}\right)$ non nécessairement maximal. Par le Lemme 2.2 on a alors

$$
l \leq \nu(8 H) N\left(r_{m}, \Gamma\left(p_{j}, p_{j+l}\right)\right) \leq \nu(8 H) N\left(\frac{r_{m}}{2 H}, \Gamma\left(p_{j}, p_{j+l}\right)\right) .
$$

Ces dernières estimations montrent bien que (3.6) a lieu avec $\tilde{H}=$ $\nu\left(4 H^{2}\right) \nu(8 H)$.

De la même façon on montre (3.5): pour pouvoir appliquer le Lemme 2.4 on se convaint qu'il existe $\tilde{H}$ dépendant que de $H$ tel que

$$
N\left(r_{M}, \Gamma\left(0, h_{M}\left(\frac{i}{M}\right)\right)\right) \leq \tilde{H} N\left(r_{M}, \Gamma\left(0, h_{m}\left(\frac{1}{m}\right)\right)\right)
$$

quand $M \geq m$ et $i / M \leq 1 / m$. On montre comme plus haut que

$$
N\left(r_{M}, \Gamma\left(0, h_{M}\left(\frac{i}{M}\right)\right)\right) \leq \nu(2 H) N\left(2 H r_{M}, \Gamma\left(0, h_{M}\left(\frac{i}{M}\right)\right)\right) \leq \nu(2 H) i .
$$

D'autre part, les Lemmes 2.2 et 2.3 font que

$$
N\left(r_{M}, \gamma\right) \leq H \nu(8 H) N\left(r_{m}, \gamma\right) N\left(r_{M}, \Gamma\left(0, h_{m}\left(\frac{1}{m}\right)\right)\right)
$$

Comme $N\left(r_{k}, \gamma\right)=k$ on a alors

$$
\frac{M}{m} \leq H \nu(8 H) N\left(r_{M}, \Gamma\left(0, h_{m}\left(\frac{1}{m}\right)\right)\right) .
$$

Or, $i / M \leq 1 / m$ et donc (3.7) est bien valable avec $\tilde{H}=H \nu(2 H) \nu(8 H)$.

En conséquence de (3.4) et (3.5) il est clair que $\left\{h_{m}\right\}$ est equicontinue. Par le Théorème d'Ascoli il est alors possible d'extraire une sous-suite de $\left\{h_{m}\right\}$ convergeant uniformément sur les compacts de $\mathbb{R}$. L'inégalité (3.4) implique que la limite $h$ satisfait l'inégalité de (I) et que $h$ est soit constante soit un homéomorphisme de $\mathbb{R}$ sur $\Gamma$. Afin de voir que $h$ n'est pas une application constante il suffit d'établir que 
$\|h(1)\|>0 ; h$ fixe 0 . Comme $\left\{h_{m}(i / m): i=0, \ldots, m\right\}$ est un $r_{m^{-}}$ ensemble de $\Gamma\left(0, h_{m}(1)\right)$ on a

$$
N\left(r_{m}, \gamma\right)=m \leq n\left(r_{m}, \Gamma\left(0, h_{m}(1)\right)\right) \leq \nu(8 H) N\left(r_{m}, \Gamma\left(0, h_{m}(1)\right)\right) .
$$

On peut alors appliquer le Lemme 2.4: il existe $d=d(H)$ tel que $\left\|h_{m}(1)\right\| \geq\|q\| / d=1 / d$. La fonction $h$ est donc bien un homéomorphisme.

b) Le cas compact: $\Gamma$ est homéomorphe à $\mathbb{S}^{1}$. Supposons que $1 \in \Gamma$. Soit $r>0$ suffisament petit et $P=\left\{p_{0}=1, p_{1}, \ldots, p_{m-1}\right\}$ ordonné $r$ espacé et maximal sur $\Gamma$ tel que $r / 2 \leq\left\|p_{m-1}-p_{0}\right\| \leq 3 r / 2$. A partir de ce choix on conclut comme dans le cas non compact.

(II) si et seulement si (I). Soit d'abord $\Gamma$ une courbe vérifiant (II) et paramétrons cette courbe à l'aide de la mesure $\omega$ : comme $\Gamma$ est un quasicercle (Lemme 2.1) c'est une courbe homéomorphe soit à $\mathbb{R}$ soit à $\mathbb{S}^{1}$. Considérons d'abord le cas non compact. Soit $p \in \Gamma$ et notons $\Gamma^{+}$et $\Gamma^{-}$les composantes connexes de $\Gamma \backslash\{p\}$. On définit $h: \mathbb{R} \longrightarrow \Gamma$ par $h(x)=q \in \overline{\Gamma^{+}}$le point avec $\omega(\Gamma(p, q))=x$ quand $x \geq 0$ et par $h(x)=q \in \Gamma^{-}$le point avec $\omega(\Gamma(p, q))=-x$ quand $x<0$. Quand $\Gamma$ est homéomorphe à $\mathbb{S}^{1}$ on note $\lambda$ la mesure de Lebesque du cercle normalisée de sorte que $\lambda\left(\mathbb{S}^{1}\right)=\omega(\Gamma)$. Pour définir $h: \mathbb{S}^{1} \longrightarrow \Gamma$ on prend $x \in \mathbb{S}^{1}, p \in \Gamma$ et on munit les deux courbes d'une orientation. Alors, l'image de $y \in \mathbb{S}^{1}$ soit $h(y)=q \in \Gamma$ le point tel que les segments joignant dans le sens positif $x$ à $y, p$ à $q$ respectivement, ont une même longueur (mesuré avec $\lambda, \omega$ respectivement).

Dans les deux cas on obtient ainsi un homéomorphisme $h: \mathbb{R}$ ou $\mathbb{S}^{1} \longrightarrow \Gamma$. Montrons pour $\Gamma$ non compact que cette paramétrisation vérifie l'inégalité de (I). Soit $h: \mathbb{R} \longrightarrow \Gamma, x, y \in \mathbb{R}$ et $s \geq t>0$. On note $p_{1}=h(x), p_{2}=h(x+t), q_{1}=h(y), q_{2}=h(y+s)$ et $R=\left\|q_{2}-q_{1}\right\|$. Soit $\left\{a_{0}, a_{1}, \ldots, a_{n}\right\}$ un $R$-ensemble de $\Gamma\left(p_{1}, p_{2}\right)$. Quand $n=1$, forcément $\left\|p_{1}-p_{2}\right\| \leq\left\|q_{1}-q_{2}\right\|$. Sinon, les hypothèses sur $\omega$ et la définition de $h$ impliquent

$$
\begin{aligned}
t=\omega\left(\Gamma\left(p_{1}, p_{2}\right)\right) & =\sum_{i=1}^{n} \omega\left(\Gamma\left(a_{i-1}, a_{i}\right)\right) \\
& \geq \frac{n-1}{A} \omega\left(\Gamma\left(q_{1}, q_{2}\right)\right) \geq \frac{n}{2 A} s \geq \frac{n}{2 A} t .
\end{aligned}
$$

On en déduit que $\left\|p_{1}-p_{2}\right\| \leq n R \leq 2 A\left\|q_{1}-q_{2}\right\|$. 
Pour la réciproque on pose $\omega(E)=\left|h^{-1}(E)\right|=\int_{h^{-1}(E)} d x$ où $E \subset \Gamma$ est un ensemble mesurable. C'est une mesure non triviale et $(\sigma-)$ finie, i.e. $\omega(\Gamma(p, q))=\omega(\Gamma(h(x+t), h(x)))=t$. Montrons qu'elle vérifie bien l'inégalité de (II). Soit $R=\left\|p_{1}-p_{2}\right\|=\left\|q_{1}-q_{2}\right\|$ avec $p_{i}, q_{i} \in \Gamma$ et soit $0<t=\omega\left(\Gamma\left(p_{1}, p_{2}\right)\right)<\omega\left(\Gamma\left(q_{1}, q_{2}\right)\right)=s$. Notons $y_{i}=h^{-1}\left(q_{i}\right)$ et $a_{j}=h\left(y_{1}+j t\right)$; on suppose que $y_{1}<y_{2}$. (I) implique $\left\|a_{i}-a_{i-1}\right\| \geq\left\|p_{2}-p_{1}\right\| / M=R / M$.

Soit $k \in \mathbb{N}$ le plus petit entier tel que $y_{1}+k t \geq y_{2}$, i.e. tel que $k t \geq s . \Gamma$ est un $K=K(M)$-quasicercle ce qui implique $\left\|a_{i}-a_{j}\right\| \geq$ $R /(K M)$ quand $i \neq j$. Par conséquent tout disque de rayon $R /(2 K M)$ contient au plus un des points $a_{i}$ et donc

$$
k \leq N\left(\frac{R}{2 K M}, \Gamma\left(q_{1}, q_{2}\right)\right) \leq \nu\left(2 K^{2} M\right) N\left(K R, \Gamma\left(q_{1}, q_{2}\right)\right)=\nu\left(2 K^{2} M\right)
$$

ce qui montre bien

$$
\omega\left(\Gamma\left(q_{1}, q_{2}\right)\right) \leq \sum_{i=1}^{k} \omega\left(\Gamma\left(a_{i-1}, a_{i}\right)\right) \leq \nu\left(2 K^{2} M\right) \omega\left(\Gamma\left(p_{1}, p_{2}\right)\right) .
$$

\section{1. $Q I$-cercles avec constante un.}

Pour terminer la démonstration du Théorème 1.1 il suffit de préciser ce qui se passe quand une des constantes est égale à un. Or, dans le cas où le groupe est 1-quasi-isométrique c'est un groupe d'isométries et donc $\Gamma$ est soit une droite soit un cercle. Si la constante de (I) est un, l'homéomorphisme $h$ est une 1-quasisymétrie et [McKV] implique que $h$ est une transformation de Möbius. Quand $\Gamma$ vérifi (II) avec constante $A=1$ il est clair que l'homéomorphisme obtenu par paramétrisation comme dans la preuve (II) implique (I) est égalément une 1-quasisymétrie. Le cas restant se trouve dans la

Proposition 3.1. Soit $\Gamma$ une courbe vérifiant (III) avec constante égale à un, alors il s'agit soit d'une droite soit d'un cercle.

La preuve de ce fait est basée sur le résultat suivant.

Lemme 3.2. Soit $\Gamma$ une courbe vérifiant (III) et soient $\gamma_{i}=\Gamma\left(p_{i}, q_{i}\right)$, $i=1, \ldots, k$, des arcs disjoints. Alors

$$
\lim _{r \rightarrow 0} \frac{N\left(r, \gamma_{1}\right)+\cdots+N\left(r, \gamma_{k}\right)}{N\left(r, \gamma_{1} \cup \cdots \cup \gamma_{k}\right)}=1
$$


Preuve. Il suffit de considérer deux arcs disjoints $\gamma_{1}$ et $\gamma_{2}$. Si $\bar{\gamma}_{1} \cap \bar{\gamma}_{2}=$ $\varnothing$ tout est clair. Sinon, $\{p\}=\bar{\gamma}_{1} \cap \bar{\gamma}_{2}$, on a toujours $N\left(r, \gamma_{1}\right)+N\left(r, \gamma_{2}\right) \geq$ $N\left(r, \gamma_{1} \cup \gamma_{2}\right)$.

Soit $\gamma_{i, r}=\gamma_{i} \backslash\left(\gamma_{i} \cap D(p, 10 H r)\right)$ pour $i=1,2$ et $r>0$ petit par rapport à diam $\gamma_{i}, i=1,2$. Puisque $\Gamma$ est un $2 H$-quasicercle (Lemme $2.1)$ on a $\left\|x_{1}-x_{2}\right\| \geq 10 H r / 2 H=5 r$ pour tout $x_{i} \in \gamma_{i, r}, i=1,2$. Les disques de rayon $r$ d'un recouvrement minimal de $\gamma_{1, r}$ sont alors disjoints des disques d'un tel recouvrement de $\gamma_{2, r}$. D'où

$$
N\left(r, \gamma_{1, r}\right)+N\left(r, \gamma_{2, r}\right) \leq N\left(r, \gamma_{1} \cup \gamma_{2}\right) \text {. }
$$

En réutilisant le fait que le disque $D(p, 10 H r)$ se couvre par au plus $\nu=\nu(10 H)$ disques de rayon $r$, on en déduit

$$
\begin{aligned}
N\left(r, \gamma_{1}\right)+N\left(r, \gamma_{2}\right) & \leq N\left(r, \gamma_{1, r}\right)+N\left(r, \gamma_{2, r}\right)+2 \nu \\
& \leq N\left(r, \gamma_{1} \cup \gamma_{2}\right)+2 \nu,
\end{aligned}
$$

ce qui achève la preuve.

Preuve De la Proposition 3.1. Il suffit de reprendre la preuve de (III) implique (I) et de voir que la constante $C_{1}(H)$ dans (3.4) peut être prise égale à un quand $H=1$. Dans ce cas, on obtient encore une 1-quasisymétrie envoyant la droite ou le cercle sur $\Gamma$.

Notons $p_{i}=h_{m}(i / m)$ et supposons qu'on puisse avoir $\left\|p_{i+k}-p_{i}\right\|>$ $\left\|p_{j+l}-p_{j}\right\|$ avec $k \leq l$. Dans ce cas il existe $x \in \Gamma\left(p_{i}, p_{i+k}\right)$ avec $\left\|x-p_{i}\right\|=\left\|p_{j+l}-p_{j}\right\|=R$. Alors, par le Lemme 3.2 et par (III) avec $H=1$

$$
\begin{aligned}
1 \geq \frac{k}{l} & =\lim _{r \rightarrow 0} \frac{\sum_{\nu=1}^{k} N\left(r, \Gamma\left(p_{i+\nu-1}, p_{i+\nu}\right)\right)}{\sum_{\nu=1}^{l} N\left(r, \Gamma\left(p_{j+\nu-1}, p_{j+\nu}\right)\right)} \\
& =\lim _{r \rightarrow 0} \frac{N\left(r, \Gamma\left(p_{i}, x\right)\right)+N\left(r, \Gamma\left(x, p_{i+k}\right)\right)}{N\left(r, \Gamma\left(p_{j}, p_{j+l}\right)\right)} .
\end{aligned}
$$

Or, quand $\left\|p_{i+k}-x\right\|>R$ il est clair que $N\left(r, \Gamma\left(x, p_{i+k}\right)\right) \geq N\left(r, \Gamma\left(p_{i}, x\right)\right)$, $r<R$. Sinon on applique le Lemme 2.3,

$$
n\left(\left\|p_{i+k}-x\right\|, \Gamma\left(p_{i}, x\right)\right) N\left(r, \Gamma\left(x, p_{i+k}\right)\right) \geq N\left(r, \Gamma\left(p_{i}, x\right)\right) .
$$

D'où, il existe $\varepsilon>0$ tel que

$$
1 \geq 1+\lim _{r \rightarrow 0} \frac{N\left(r, \Gamma\left(x, p_{i+k}\right)\right)}{N\left(r, \Gamma\left(p_{j}, p_{j+l}\right)\right)} \geq 1+\varepsilon \lim _{r \rightarrow 0} \frac{N\left(r, \Gamma\left(p_{i}, x\right)\right)}{N\left(r, \Gamma\left(p_{j}, p_{j+l}\right)\right)}=1+\varepsilon,
$$

ce qui est impossible. 


\section{Les $Q I$-cercles et la géométrie fractale.}

Comme on l'a remarqué dans l'introduction, les "quasi-self-similar" cercles sont des $Q I$-cercles. Quasi-auto-similarité veut dire que des petits morceaux d'une courbe s'agrandissent par une similitude en des arcs d'une taille standart, lesquels se plongent quasi-isométriquement dans la courbe elle même. Une conséquence de ceci est que les sous-arcs d'une même taille sont quasi-isométriques; $c f$. (1.1). Cette dernière propriété est aussi valable pour les $Q I$-cercles. On peut même dire qu'elle les caractérise. Par contre, il n'y a plus de rapport entre des sous-arcs de différentes tailles.

Cet affaiblissement des propriétés fait que les résultats des "quasiself-similar" cercles ne se transmettent pas directement à des $Q I$-cercles. Néanmoins, on peut montrer que pour un $Q I$-cercle la dimension de Hausdorff coïncide avec la "lower Box-dimension". En plus il est possible d'étendre le théorème de Falconer et Marsh [FM] à des $Q I$-cercles.

Pour pouvoir faire ceci nous aurons besoin de mieux connaître le comportement de certaines mesures sur un $Q I$-cercle $\Gamma$. On lui associe d'abord une fonction $\rho$, appelée fonction de dimension canonique, de la façon suivante: quand $\Gamma$ est compact on pose $\rho(r)=1 / N(r, \Gamma)$, $0<r \leq \operatorname{diam} \Gamma$. Sinon on choisit $\gamma=\Gamma(a, b)$ un sous-arc quelconque de $\Gamma$ avec $\|a-b\|=1$ et $\gamma_{r}=\Gamma(a, x) \supset \gamma$ avec $\|a-x\|=r$ ainsi que $\|a-y\|<r$ pour tout $y \in \Gamma(b, x)$. Alors, on définit maintenant $\rho(r)=1 / N(r, \gamma)$ pour $0<r \leq 1$ et $\rho(r)=N\left(1, \gamma_{r}\right)$ pour $r>1$.

Lemme 4.1. Sur un QI-cercle $\Gamma$ il existe une mesure $\omega$ et des constantes $C_{3}$ et $C_{4}$ telles que

$$
\frac{1}{C_{3}} \leq \frac{\omega(\Gamma(p, q))}{\rho(\|p-q\|)} \leq C_{3}, \quad \text { pour tous } p, q \in \Gamma, p \neq q
$$

et

$$
\omega(D(x, r)) \leq C_{4} \rho(r), \quad \text { pour tous } x \in \mathbb{R}^{2} \text { et } r>0
$$

Preuve. Soit $\omega$ la mesure de (II) du Théorème 1.1 normalisée par $\omega(\Gamma)=1$ quand $\Gamma$ est compact et par $\omega(\gamma)=1$ sinon, $\gamma$ étant l'arc de la définition de $\rho$.

Soit $\Gamma$ non compact et considérons d'abord le cas $r=\|p-q\| \leq 1$, $p, q \in \Gamma$. Notons $\left\{x_{0}, \ldots, x_{n}\right\}$ un $r$-ensemble de $\gamma$. Alors l'inégalité de 
(II) implique

$$
1=\omega(\gamma)=\sum_{i=1}^{n} \omega\left(\Gamma\left(x_{i-1}, x_{i}\right)\right) \leq A n \omega(\Gamma(p, q))
$$

et aussi $n \omega(\Gamma(p, q)) / 2 \leq A$. L'inégalité (4.8) en résulte immédiatement puisque les nombres $n$ et $N(r, \gamma)$ sont équivalents (Lemme 2.2).

Quand $r=\|p-q\|>1$ on choisit $\left\{x_{0}, \ldots, x_{n}\right\}$ un 1-ensemble de $\gamma_{r}$ et on applique encore l'inégalité de (II):

$$
\omega(\Gamma(p, q)) \leq A \omega\left(\gamma_{r}\right)=A \sum_{i=1}^{n} \omega\left(\Gamma\left(x_{i-1}, x_{i}\right)\right) \leq A^{2} n \omega(\gamma)=A^{2} n
$$

et $A^{2} \omega(\Gamma(p, q)) \geq n / 2$. Il suffit encore d'appliquer le Lemme 2.2 pour en déduire (4.8).

Quand $\Gamma$ est compact on procède de la même façon.

De ces estimations on déduit (4.9). Effectivement, puisque $\Gamma$ est un $2 H$-quasicercle, avec $H$ la constante de (III), l'ensemble $\Gamma \cap D(x, 5 H r)$ contient un arc $\sigma=\Gamma(u, v), u, v \in \partial D(x, 5 H r)$, contenant l'ensemble $\Gamma \cap D(x, r)$. D'où $\omega(D(x, r))=\omega(\Gamma \cap D(x, r)) \leq \omega(\sigma)$ et par (4.8) ceci devient $\omega(D(x, r)) \leq C_{3} \rho(\|u-v\|)$. On en déduit (4.9) puisque $\|u-v\| \leq 10 \mathrm{Hr}$.

La fonction $\rho$ définie avant le lemme précédent est croissante et elle vérifie $\lim _{r \rightarrow 0} \rho(r)=0$. A une telle fonction on peut associer la mesure de Hausdorff suivante

$$
m_{\rho}(E)=\lim _{r \rightarrow 0} \inf \left\{\sum_{i=1}^{\infty} \rho\left(r_{i}\right): E \subset \bigcup_{i=1}^{\infty} D\left(x_{i}, r_{i}\right) \text { avec } r_{i}<r\right\}
$$

pour $E \subset \Gamma$.

Lemme 4.2. Soit $\Gamma$ un $Q I$-cercle, $\rho$ sa fonction de dimension canonique et $m_{\rho}$ la mesure de Hausdorff associée. Alors, il existe une constante $C_{5}$ telle que

$$
\frac{1}{C_{5}} \rho(\|p-q\|) \leq m_{\rho}(\Gamma(p, q)) \leq C_{5} \rho(\|p-q\|), \quad \text { pour tous } p, q \in \Gamma \text {. }
$$


Preuve. L'inégalité de gauche est une conséquence du Lemme 4.1 et du lemme de Frostman. Effectivement, si $\Gamma(p, q) \subset \bigcup_{i=1}^{\infty} D\left(x_{i}, r_{i}\right)$ alors

$$
\omega(\Gamma(p, q)) \leq \sum_{i=1}^{\infty} \omega\left(D\left(x_{i}, r_{i}\right)\right) \leq C_{4} \sum_{i=1}^{\infty} \rho\left(r_{i}\right)
$$

et donc $C_{4} C_{3} m_{\rho}(\Gamma(p, q)) \geq \rho(\|p-q\|)$.

Dans la preuve de l'autre inégalité on fait appel aux Lemmes 2.2 et 2.3. Quand $R=\|p-q\|>1$ alors

$$
\begin{aligned}
m_{\rho}(\Gamma(p, q)) & \leq \liminf _{r \rightarrow 0} \frac{N(r, \Gamma(p, q))}{N(r, \gamma)} \\
& \leq H n(1, \Gamma(p, q)) \\
& \leq H^{2} \nu(8 H) \rho(\|p-q\|)
\end{aligned}
$$

et quand $R=\|p-q\| \leq 1$ on a

$$
m_{\rho}(\Gamma(p, q)) \leq \liminf _{r \rightarrow 0} \frac{N(r, \Gamma(p, q))}{N(r, \gamma)} \leq \frac{1}{\eta(H)} \frac{1}{n(R, \gamma)} \leq \frac{\rho(\|p-q\|)}{\eta(H)}
$$

\subsection{Les $Q I$-cercles et les différentes dimensions.}

Connaître le lien entre les différentes notions de dimensions a des avantages pratiques. Par exemple, la simple définition des "Box-dimensions" fait qu'elles s'évaluent facilement alors que l'estimation de la dimension de Hausdorff est souvent laborieuse et difficile.

Précisons ces termes pour un compact $K \subset \mathbb{R}^{2}$. Sa dimension de Hausdorff est $\operatorname{Hdim}(K)=\sup \left\{\delta>0: m_{r^{6}}(K)=+\infty\right\} ; m_{r^{6}}$ est la mesure de Hausdorff de dimension $\delta$. Les "Box-dimensions" sont données par

$$
\begin{aligned}
& \underline{\operatorname{Bdim}}(K)=\liminf _{r \rightarrow 0} \frac{\log N(r, K)}{-\log r}, \\
& \overline{\operatorname{Bdim}}(K)=\limsup _{r \rightarrow 0} \frac{\log N(r, K)}{-\log r} .
\end{aligned}
$$

On a toujours $\operatorname{Hdim}(K) \leq \underline{\operatorname{Bdim}}(K) \leq \overline{\operatorname{Bdim}}(K)$ et pour les "quasiself-similar" cercles ces trois nombres coïncident. 
Proposition 4.3. Un QI-cercle $\Gamma$ a toujours la dimension de Hausdorff égale à la "lower Box-dimension": $\operatorname{Hdim}(\Gamma)=\underline{\operatorname{Bdim}}(\Gamma) . \quad P a r$ contre, il existe des exemples pour lesquels les différentes "Box-dimensions" sont distinctes: $\underline{\mathrm{Bdim}}(\Gamma)<\overline{\mathrm{Bdim}}(\Gamma)$.

Preuve DE $\operatorname{Hdim}(\Gamma)=\underline{\mathrm{Bdim}}(\Gamma)$. Il est suffisant de considérer $\gamma \subset \Gamma$ un arc quelconque puisque sur $\Gamma$ agit un groupe quasi-isométrique transitif et les dimensions sont invariantes par quasi-isométrie. Prenons alors pour $\gamma$ l'arc de la définition de la fonction de dimension canonique $\rho$ quand $\Gamma$ n'est pas compact et $\gamma=\Gamma$ sinon.

Pour tout

$$
\beta<\delta=\underline{\operatorname{Bdim}}(\gamma)=\liminf _{r \rightarrow 0} \frac{\log \rho(r)}{\log r}
$$

on a $\lim _{r \rightarrow 0} \rho(r) / r^{\beta}=0$. Comme $m_{\rho}(\gamma)>0$ (Lemme 4.2) il suit que $m_{r^{\beta}}(\gamma)=+\infty$.

\section{Un exemple d'un $Q I$-cercle ayant différents "upper" et "lower} Box-dimension".

Utilisons la construction du "snowflake" et notons $T_{a, b}$ la transformation qui associe à un intervalle $I=[a, b]$ la première iterée du snowflake avec les extrémités $a$ et $b$. Plus précisement, soient $a=$ $\left(a_{1}, a_{2}\right), b=\left(b_{1}, b_{2}\right)$ ainsi que

$$
x_{1}=a+\frac{b-a}{3}, \quad x_{2}=\frac{a+b}{2}+\left(b_{2}-a_{2}, a_{1}-b_{1}\right) \frac{1}{2 \sqrt{3}}
$$

et

$$
x_{3}=a+\frac{2(b-a)}{3} .
$$

On associe à ces points quatre similitudes contractantes $T_{i}, i=1, \ldots, 4$, envoyant $\overrightarrow{a b}$ sur $\overrightarrow{a x_{1}}, \overrightarrow{x_{1} x_{2}}, \overrightarrow{x_{2} x_{3}}, \overrightarrow{x_{3} b}$, respectivement. Notons

$$
T_{a, b}(K)=\bigcup_{i=1}^{4} T_{i}(K), \quad K \text { compact de } \mathbb{R}^{2} .
$$

Soit $J_{0}=[a, b]$ avec $\|a-b\|=1$ et $J_{1}=T_{a, b}\left(J_{0}\right) . \quad J_{1}$ consiste en quatre côtés de longueur $1 / 3$, ce qui correspond à une dimension $\delta_{1}=\log 4 / \log 3$. Soit $0<\varepsilon<\left(\delta_{1}-1\right) / 4$. Il existe $k_{1} \in \mathbb{N}$ tel que

$$
4 k_{1}\left(\frac{1}{3 k_{1}}\right)^{\delta}=1, \quad \text { avec } 1 \leq \delta \leq 1+\varepsilon .
$$


En fait, on saute $k_{1}$ itérations de $T_{a, b}$ pour ramener la dimension proche de un. Partageons alors $J_{1}$ en $4 k_{1}\left[a_{i}, b_{i}\right]$ de longueur $1 /\left(3 k_{1}\right): J_{1}=$ $\cup_{i=1}^{4} k_{1}\left[a_{i}, b_{i}\right]$. Maintenant on va effectuer $k_{2}$ itérations de $T_{a_{i} b_{i}}$ sur les intervalles $\left[a_{i}, b_{i}\right]$. Soit donc

$$
J_{2}=\bigcup_{i=1}^{4 k_{1}} T_{a_{i} b_{i}}^{k_{2}}\left(\left[a_{i}, b_{i}\right]\right)
$$

où $k_{2}$ est choisi de sorte que

$$
4^{k_{2}} 4 k_{1}\left(\frac{1}{3^{k_{2}}} \frac{1}{3 k_{1}}\right)^{\delta}=1,
$$

avec cette fois ci $\delta_{1}-\varepsilon<\delta \leq \delta_{1}$. Les prochaines $k_{3}$ itérations de $T_{a_{i} b_{i}}$ on les saute pour avoir de nouveau une dimension proche de un et ainsi de suite.

Soit $\Gamma(a, b)$ la limite de ce procédé. L'exemple cherché est

$$
\Gamma=\Gamma((0,0),(1,0)) \cup \Gamma\left((0,0),\left(\frac{1}{2}, \frac{\sqrt{3}}{2}\right)\right) \cup \Gamma\left(\left(\frac{1}{2}, \frac{\sqrt{3}}{2}\right),(1,0)\right) .
$$

Clairement, $\Gamma$ est un $Q I$-cercle le critère géométrique (III) étant vérifié et on a

$$
\underline{\mathrm{Bdim}}(\Gamma)<\overline{\mathrm{Bdim}}(\Gamma) .
$$

\subsection{Classification des $Q I$-cercles par quasi-isométrie.}

$\mathrm{Si}$ on veut établir un analogue du théorème de Falconer et Marsh [FM] pour les $Q I$-cercles on est amené à prendre une notion plus forte que seulement la dimension, à cause de l'exemple du paragraphe précédent: ce sont les classes de fonctions de dimension.

On appelle une fonction $\rho: \mathbb{R}^{+} \longrightarrow \mathbb{R}^{+}$croissante et vérifiant $\lim _{r \rightarrow 0} \rho(r)=0$ ainsi que $\lim _{r \rightarrow \infty} \rho(r)=+\infty$ fonction de dimension. Remarquons que pour les courbes compactes seul le comportement de $\rho$ au voisinage de 0 est important. On se contente alors dans ce cas de définir la fonction de dimension au voisinage de 0 . Deux fonctions de dimension $\rho_{1}, \rho_{2}$ sont équivalentes s'il existe $\alpha, \beta>0$ tels que

$$
\alpha \leq \frac{\rho_{1}(r)}{\rho_{2}(r)} \leq \beta, \quad \text { pour tout } r>0 .
$$


Au début de ce paragraphe nous avons associé à un $Q I$-cercle $\Gamma$ une fonction de dimension canonique $\rho$. L'ensemble de fonctions de dimensions équivalentes à ce $\rho$ est appelé la classe de fonctions de dimension associée à $\Gamma$.

Théorème 4.4. Soient $\Gamma_{1}$ et $\Gamma_{2}$ deux $Q I$-cercles, tout deux compacts ou non. Il existe $\Phi: \mathbb{R}^{2} \longrightarrow \mathbb{R}^{2}$ une quasi-isométrie identifiant les courbes, $\Phi\left(\Gamma_{1}\right)=\Gamma_{2}$, si et seulement si $\Gamma_{1}$ et $\Gamma_{2}$ ont la même classe de fonctions de dimension.

Remarque. Ce théorème contient le résultat de Falconer et Marsh $[F M]$ : si $\Gamma$ est un "quasi-self-similar" cercle, sa classe de fonctions de dimension est celle qui contient la fonction constante $\delta(r)=\operatorname{dim} \Gamma$. D'où, pour deux telles courbes $\Gamma_{1}$ et $\Gamma_{2}$ il existe une quasi-isométrie $\varphi: \Gamma_{1} \longrightarrow \Gamma_{2}$ si et seulement si les deux courbes ont la même dimension.

Preuve. Montrons l'existence de la quasi-isométrie $\Phi$ sous l'hypothèse que les $\Gamma_{i}$ ont la même classe de fonctions de dimension. On note $\rho_{1}, \rho_{2}$ les fonctions de dimension canoniques de $\Gamma_{1}, \Gamma_{2}$ respectivement et $m_{\rho_{i}}$ les mesures de Hausdorff associées. Ces mesures permettent de paramétrer les courbes $\Gamma_{i}$; $c f$. la preuve (II) implique (I). Notons $h_{i}: \mathbb{R}$ ou $\mathbb{S}^{1} \longrightarrow \Gamma_{i}, i=1,2$, ces paramétrisations. On montre que $\varphi=h_{2} \circ h_{1}^{-1}: \Gamma_{1} \longrightarrow \Gamma_{2}$ est une quasi-isométrie.

Par le Lemme 4.2 il existe $C_{5} \geq 1$ tel que

$$
\frac{\rho_{i}(\|u-v\|)}{C_{5}} \leq m_{\rho_{i}}\left(\Gamma_{i}(u, v)\right) \leq C_{5} \rho_{i}(\|u-v\|), \quad u, v \in \Gamma_{i} .
$$

Si $p_{1}, p_{2} \in \Gamma_{1}$ et si $q_{i}=\varphi\left(p_{i}\right) \in \Gamma_{2}, i=1,2$, alors $m_{\rho_{1}}\left(\Gamma_{1}\left(p_{1}, p_{2}\right)\right)=$ $m_{\rho_{2}}\left(\Gamma_{2}\left(q_{1}, q_{2}\right)\right)$ et donc

$$
\frac{1}{C_{5}^{2}} \leq \frac{\rho_{1}\left(\left\|p_{1}-p_{2}\right\|\right)}{\rho_{2}\left(\left\|q_{1}-q_{2}\right\|\right)} \leq C_{5}^{2} .
$$

Par l'équivalence de $\rho_{1}$ et $\rho_{2}$ il existe $\alpha, \beta>0$ telles que

$$
\frac{\beta}{C_{5}^{2}} \leq \frac{\rho_{1}\left(\left\|p_{1}-p_{2}\right\|\right)}{\rho_{1}\left(\left\|q_{1}-q_{2}\right\|\right)} \leq \alpha C_{5}^{2}
$$

De cette condition il est facile à voir que $\varphi$ est une quasi-isométrie. Il suffit d'expliciter $\rho_{1}(r)$ dans les différents cas et d'utiliser le Lemme 2.3. 
On peut prolonger $\varphi$ en une quasi-isométrie $\Phi$ du plan grâce à un résultat de Gehring [Ge1]. Une autre possibilité est de montrer, comme dans la preuve "(I) implique $\Gamma$ est un $Q I$-cercle", que $\Phi=H_{2} \circ H_{1}^{-1}$ : $\mathbb{R}^{2} \rightarrow \mathbb{R}^{2}$, avec $H_{i}$ les extensions quasiconformes de Beurling-AhlforsTukia des quasisymétries $h_{i}$, est une quasi-isométrie du plan.

Soit maintenant $\Phi$ une $L$-quasi-isométrie de $\mathbb{R}^{2}$ telle que $\Phi\left(\Gamma_{1}\right)=$ $\Gamma_{2}$ et notons encore $\rho_{1}, \rho_{2}$ les fonctions de dimensions canoniques de $\Gamma_{1}, \Gamma_{2}$ respectivement. On doit montrer qu'elles sont équivalentes.

Considérons d'abord le cas $\Gamma_{1}$ et $\Gamma_{2}$ compact. Si $D\left(x_{1}, r\right), \ldots$, $D\left(x_{N}, r\right)$ est un recouvrement minimal de $\Gamma_{1}$, alors les ensembles $E_{i}=\Phi\left(D\left(x_{i}, r\right)\right)$ couvrent $\Gamma_{2}$ et $E_{i} \subset D\left(\Phi\left(x_{i}\right), L r\right)$. D'où et avec (2.2) il est clair que $N\left(r, \Gamma_{1}\right) \geq N\left(L r, \Gamma_{2}\right) \geq N\left(r, \Gamma_{2}\right) / \nu(L)$. Par symétrie du problème il en résulte $1 / \nu(L) \leq \rho_{1}(r) / \rho_{2}(r) \leq \nu(L)$.

Quand les $\Gamma_{i}$ ne sont pas compacts on doit montrer qu'il existe $C \geq 1$ tel que

$$
\frac{1}{C} \leq \frac{N\left(r, \gamma_{1}\right)}{N\left(r, \gamma_{2}\right)} \leq C, \quad \text { et } \quad \frac{1}{C} \leq \frac{N\left(1, \gamma_{1, r}\right)}{N\left(1, \gamma_{2, r}\right)} \leq C
$$

où les arcs viennent de la définition des $\rho_{i}$. Ce qui compte est que les extrémités des $\gamma_{i}$ et des $\gamma_{i, r}$ sont à distance égale. D'où, la preuve de ceci est exactement le contenue de la preuve " $Q I$-cercle implique (III)". Le rôle des $g_{t}$ dans cette preuve prend ici $g_{2, t} \circ \Phi$ où $G_{2}=\left\{g_{2, t}: t \in \mathbb{R}\right\}$ est le groupe quasi-isométrique de $\Gamma_{2}$.

\section{References.}

[Bo] Bowen, R., Hausdorff dimension of quasi-circles. Publ. Math. IHES 50, 259-273.

[Fa] Falconer, K. J., Fractal Geometry. Mathematical Foundations and Applications, John Wiley, 1990.

[FM] Falconer, K. J. and Marsh, T. D., Classification of quasi-circles by Hausdorff-dimension. Nonlinearity 2 (1989), 489-493.

[Ge1] Gehring, F. W., Injectivity of local quasi-isometries. Comm. Math. Helv. 57 (1982), 202-220.

[Ge2] Gehring, F. W., Characteristic properties of quasidiscs. Les presses de l'Université de Montréal (1982).

[Gr] Greenberg, L., Discrete subgroups of the Lorentz group. Math. Scandinavica 10 (1962), 85-107. 
[McKV] McKemie, M. J. and Vaaler, J. D., Weakly quasisymmetric embeddings of $\mathbb{R}$ into $\mathbb{C}$. Ann. Acad. Sci. Fenn. Ser. A.I. 12 (1987), 163-170.

[Su1] Sullivan, D., On the ergodic theory at infinity of an arbitrary discrete group of hyperbolic motions. Ann. of Math. Stud. 97 (1981), 465-496.

[Su2] Sullivan, D., Conformal dynamical systems. Lecture Notes in Math. 1007 (1983), 725-752.

[T1] Tukia, P., On two-dimensional quasiconformal groups. Ann. Acad. Sci. Fenn. Ser. A.I. 5 (1980), 73-80.

[T2] Tukia, P., A quasiconformal group not isomorphic to a Möbius group. Ann. Acad. Sci. Fenn. Ser. A.I. 6 (1981), 149-160.

[T3] Tukia, P., Extension of quasisymmetric and Lipschitz embeddings of the real line into the plane. Ann. Acad. Sci. Fenn. Ser. A.I. 6 (1981), 89-94.

Recibido: 19 de mayo de 1.993

Revisión: 8 de abril de 1.994

Volker Mayer

CEREMAB. Université de Bordeaux I

351 , Cours de la Libération

F-33405 Talence Cedex, FRANCE

and

U.R.A. 751 du C.N.R.S. "GAT"

U.F.R. de Mathématiques Pures et Appliquées

Université des Sciences et Technologies de Lille 59655 Villeneuve d'Ascq Cedex, FRANCE mayer@gat .univ-lille1.fr 